Consistency between Angular

Distributions and Integral Quantities

Calculated with the Nuclear Ramsauer Model

F. S. Dietrich

December 21, 2004 
This document was prepared as an account of work sponsored by an agency of the United States Government. Neither the United States Government nor the University of California nor any of their employees, makes any warranty, express or implied, or assumes any legal liability or responsibility for the accuracy, completeness, or usefulness of any information, apparatus, product, or process disclosed, or represents that its use would not infringe privately owned rights. Reference herein to any specific commercial product, process, or service by trade name, trademark, manufacturer, or otherwise, does not necessarily constitute or imply its endorsement, recommendation, or favoring by the United States Government or the University of California. The views and opinions of authors expressed herein do not necessarily state or reflect those of the United States Government or the University of California, and shall not be used for advertising or product endorsement purposes.

This work was performed under the auspices of the U.S. Department of Energy by University of California, Lawrence Livermore National Laboratory under Contract W-7405-Eng-48. 


\title{
Consistency between Angular Distributions and Integral Quantities Calculated with the Nuclear Ramsauer Model
}

\author{
Frank S. Dietrich \\ Lawrence Livermore National Laboratory, Livermore, CA 94550
}

December 20, 2004

\begin{abstract}
This report reviews the connection between the exact expression for the scattering amplitude and its approximation by the nuclear Ramsauer model. This approximation is well defined only for integral values of $\mathrm{kR}$, the product of the neutron wave number and a nuclear radius parameter. Using the model between these integral values requires the introduction of an interpolation scheme. We show the effects on physical observables of adding an extra term to the scattering amplitude. By choosing the functional form of this term it is possible to obtain smooth behavior with energy of some of the physical observables, but not all.
\end{abstract}




\section{General partial wave expansion}

The fundamental quantity that determines scattering observables is the scattering amplitude. Since the predictions of the nuclear Ramsauer effect are most easily seen by making approximations to the scattering amplitude, we begin with the complete expressions for the scattering amplitude and for the quantities derived from it.

Assume a spinless target and a spinless, uncharged projectile. Then the general expression for the scattering amplitude is

$$
f(\theta)=\frac{1}{k} \sum_{l=0}^{\infty}(2 l+1) e^{i \delta_{l}} \sin \delta_{l} P_{l}(\cos \theta),
$$

where $\delta_{l}$ is a complex phase shift that depends on partial wave $l$. We define an $S$ matrix element $S_{l}$ and parameterize it via either the complex phase shift $\delta_{l}$ or the real parameters $\alpha_{l}$ and $\beta_{l}$ by

$$
S_{l}=e^{2 i \delta_{l}}=\alpha_{l} e^{i \beta_{l}} .
$$

Then we can write the scattering amplitude as

$$
\begin{aligned}
f(\theta) & =\frac{i}{2 k} \sum_{l=0}^{\infty}(2 l+1)\left(1-S_{l}\right) P_{l}(\cos \theta) \\
& =\frac{i}{2 k} \sum_{l=0}^{\infty}(2 l+1)\left(1-\alpha_{l} e^{i \beta_{l}}\right) P_{l}(\cos \theta) .
\end{aligned}
$$

The forward scattering amplitude is

$$
f(0)=\frac{i}{2 k} \sum_{l=0}^{\infty}(2 l+1)\left(1-S_{l}\right) .
$$

By inserting this in the optical theorem $\sigma_{\text {tot }}=(4 \pi / k) \operatorname{Im} f(0)$ we obtain the total cross section

$$
\sigma_{t o t}=2 \pi \lambda^{2} \sum_{l=0}^{\infty}(2 l+1)\left(1-\operatorname{Re} S_{l}\right) .
$$

The differential elastic cross section is

$$
\frac{d \sigma_{\text {elas }}}{d \Omega}=|f(\theta)|^{2}=\frac{1}{4} \lambda^{2}\left|\sum_{l=0}^{\infty}(2 l+1)\left(1-S_{l}\right) P_{l}(\cos \theta)\right|^{2}
$$

which can easily be integrated over solid angle to yield the complete elastic cross section

$$
\sigma_{\text {elas }}=\pi \lambda^{2} \sum_{l=0}^{\infty}(2 l+1)\left|1-S_{l}\right|^{2} .
$$

Subtracting this from the total cross section leads to the reaction (nonelastic) cross section

$$
\sigma_{\text {reac }}=\pi \lambda^{2} \sum_{l=0}^{\infty}(2 l+1)\left(1-\left|S_{l}\right|^{2}\right) .
$$


In terms of $\alpha_{l}$ and $\beta_{l}$ the total, elastic, and reaction cross sections are

$$
\begin{aligned}
\sigma_{\text {tot }} & =2 \pi \lambda^{2} \sum_{l=0}^{\infty}(2 l+1)\left(1-\alpha_{l} \cos \beta_{l}\right), \\
\sigma_{\text {elas }} & =\pi \lambda^{2} \sum_{l=0}^{\infty}(2 l+1)\left(1+\alpha_{l}^{2}-2 \alpha_{l} \cos \beta_{l}\right), \\
\sigma_{\text {reac }} & =\pi \lambda^{2} \sum_{l=0}^{\infty}(2 l+1)\left(1-\alpha_{l}^{2}\right) .
\end{aligned}
$$

Additional useful quantities are the true zero-degree cross section $\sigma_{0}$ and its Wick's limit approximation $\sigma_{0}^{W}$,

$$
\begin{aligned}
\sigma_{0} & =[\operatorname{Re} f(0)]^{2}+[\operatorname{Im} f(0)]^{2} \\
\sigma_{0}^{W} & =[\operatorname{Im} f(0)]^{2}=\left(k \sigma_{\text {tot }} / 4 \pi\right)^{2}
\end{aligned}
$$

which we evaluate using the expressions

$$
\begin{aligned}
& \operatorname{Re} f(0)=\frac{1}{2 k} \sum_{l=0}^{\infty}(2 l+1)\left(1-\operatorname{Re} S_{l}\right) \\
& \operatorname{Im} f(0)=\frac{1}{2 k} \sum_{l=0}^{\infty}(2 l+1) \operatorname{Im} S_{l}
\end{aligned}
$$

which in terms of the $\alpha_{l}$ and $\beta_{l}$ are

$$
\begin{aligned}
\operatorname{Re} f(0) & =\frac{1}{2 k} \sum_{l=0}^{\infty}(2 l+1)\left(1-\alpha_{l} \cos \beta_{l}\right), \\
\operatorname{Im} f(0) & =\frac{1}{2 k} \sum_{l=0}^{\infty}(2 l+1) \alpha_{l} \sin \beta_{l} .
\end{aligned}
$$

We also define a fractional deviation of the true zero-degree cross section from Wick's limit by

$$
\eta=\left(\sigma_{0}-\sigma_{0}^{W}\right) / \sigma_{0}^{W} .
$$

Finally, we show the expression for $\bar{\mu}$, the average of the cosine of the scattering angle. The quantity $\bar{\mu}$ is defined as

$$
\bar{\mu}=\frac{1}{\sigma_{\text {elas }}} \int d \Omega \cos \theta \frac{d \sigma(\theta)}{d \Omega} .
$$

By using the recurrence relation for Legendre polynomials (see, for example, Eq. (2.5.20) in Edmonds [1]),

$$
(l+1) P_{l+1}(\cos \theta)-(2 l+1) \cos \theta P_{l}(\cos \theta)+l P_{l-1}(\cos \theta)=0,
$$

together with the orthonormality relation

$$
\frac{1}{4 \pi} \int d \Omega \sqrt{\left(2 l^{\prime}+1\right)(2 l+1)} P_{l^{\prime}}(\cos \theta) P_{l}(\cos \theta)=\delta_{l^{\prime} l},
$$

it is easy to find

$$
\sigma_{\text {elas }} \bar{\mu}=\pi \lambda^{2} \sum_{l=0}^{\infty}\left\{l\left(1-S_{l-1}^{*}\right)\left(1-S_{l}\right)+(l+1)\left(1-S_{l+1}^{*}\right)\left(1-S_{l}\right)\right\},
$$

where for $l=0$ the terms with index $l-1$ in this expression and in Eq. 20 should be omitted. 


\section{The Ramsauer approximation}

The Ramsauer approximation is obtained very simply from the general expression for the scattering amplitude by assuming the complex phase shifts (or, equivalently, the $\alpha_{l}$ and $\beta_{l}$ ) are independent of $l$ up to and including partial wave $l_{\text {max }}$, and are zero for higher partial waves. Thus the Ramsauer-model scattering amplitude is

$$
f(\theta)=\frac{i}{2 k}\left(1-\alpha e^{i \beta}\right) \sum_{l=0}^{l_{\max }}(2 l+1) P_{l}(\cos \theta) .
$$

We choose $l_{\max }$ by the condition $l_{\max }=k R$, where $R$ is a radius parameter. For purposes of this derivation we assume that, for a given value of $R, k$ is restricted to a discrete range of values so that $l_{\max }$ is an integer. This is not a serious limitation for angle-independent quantities. In the following we make frequent use of the expression

$$
\pi \lambda^{2} \sum_{l=0}^{l_{\max }}(2 l+1)=\pi \lambda^{2}\left(l_{\text {max }}+1\right)^{2}=\pi(R+\lambda)^{2} .
$$

The Ramsauer approximation yields the following expressions for the total, elastic, and reaction cross sections:

$$
\begin{aligned}
\sigma_{\text {tot }} & =2 \pi(R+\lambda)^{2}(1-\alpha \cos \beta), \\
\sigma_{\text {elas }} & =\pi(R+\lambda)^{2}\left|1-\alpha e^{i \beta}\right|^{2} \\
& =\pi(R+\lambda)^{2}\left(1+\alpha^{2}-2 \alpha \cos \beta\right), \\
\sigma_{\text {reac }} & =\pi(R+\lambda)^{2}\left(1-\alpha^{2}\right) .
\end{aligned}
$$

The forward scattering amplitude is

$$
\begin{aligned}
f(0) & =\frac{i}{2 k}\left(1-\alpha e^{i \beta}\right) \sum_{l=0}^{l_{\max }}(2 l+1) \\
& =\frac{1}{2} i k(R+\lambda)^{2}\left(1-\alpha e^{i \beta}\right),
\end{aligned}
$$

which has the real and imaginary parts

$$
\begin{aligned}
& \operatorname{Re} f(0)=\frac{1}{2} k(R+\lambda)^{2} \alpha \sin \beta, \\
& \operatorname{Im} f(0)=\frac{1}{2} k(R+\lambda)^{2}(1-\alpha \cos \beta) .
\end{aligned}
$$

From these we obtain the zero-degree differential cross section

$$
\sigma_{0}=|f(0)|^{2}=\frac{1}{4} k^{2}(R+\lambda)^{4}\left(1+\alpha^{2}-2 \alpha \cos \beta\right) ;
$$

its Wick's limit approximation

$$
\sigma_{0}^{W}=[\operatorname{Im} f(0)]^{2}=\frac{1}{4} k^{2}(R+\lambda)^{4}(1-\alpha \cos \beta)^{2} ;
$$

the difference between these,

$$
\sigma_{0}-\sigma_{0}^{W}=[\operatorname{Re} f(0)]^{2}=\frac{1}{4} k^{2}(R+\lambda)^{4} \alpha^{2} \sin ^{2} \beta ;
$$


and the fractional deviation

$$
\eta=\frac{\sigma_{0}-\sigma_{0}^{W}}{\sigma_{0}^{W}}=\frac{\alpha^{2} \sin ^{2} \beta}{(1-\alpha \cos \beta)^{2}} .
$$

We determine the Ramsauer-model value for the average cosine of the scattering angle by applying the Ramsauer approximation to Eq. 22. We begin by including terms in the sum through $l=l_{\max }-1$ and show the $l=l_{\max }$ term explicitly. For this last term, the second term within the brackets in Eq. 22 must be dropped because it contains the factor $\left(1-S_{l_{m a x}+1}^{*}\right)$, which vanishes because of the sharp-cutoff approximation. Thus

$$
\begin{aligned}
\sigma_{\text {elas }} \bar{\mu} & =\pi \lambda^{2}\left|1-\alpha e^{i \beta}\right|^{2}\left\{\left[\sum_{l=0}^{l_{\max }-1}(2 l+1)\right]+l_{\max }\right\} \\
& =\pi \lambda^{2}\left|1-\alpha e^{i \beta}\right|^{2}\left\{\left[\sum_{l=0}^{l_{\max }}(2 l+1)\right]-\left(l_{\max }+1\right)\right\} \\
& =\pi \lambda^{2}\left|1-\alpha e^{i \beta}\right|^{2}\left\{\left(l_{\max }+1\right)^{2}-\left(l_{\max }+1\right)\right\} \\
& =\pi \lambda^{2}\left|1-\alpha e^{i \beta}\right|^{2}\left(l_{\max }+1\right)^{2}\left(1-\frac{1}{l_{\max }+1}\right) \\
& =\pi(R+\lambda)^{2}\left|1-\alpha e^{i \beta}\right|^{2}\left(1-\frac{1}{k R+1}\right) .
\end{aligned}
$$

We recognize $\sigma_{\text {elas }}$ in the right hand side of the last expression, and cancel it to get

$$
\bar{\mu}=1-\frac{1}{k R+1}
$$

or equivalently an expression that emphasizes scattering away from forward angles,

$$
1-\bar{\mu}=\frac{1}{k R+1} \text {. }
$$

The last two expressions exhibit universal behavior in the sense that the only dependence on nuclear parameters is in the radius $R$. In particular, there is no dependence on $\alpha$ and $\beta$.

In all of the above expressions, we may interpolate between the discrete values of $k$ by making the simplest choice, namely that $k$ can treated as a continuous variable. This is also intuitively appealing since it should be expected that the physical observables behave smoothly with energy.

In the Ramsauer approximation, the elastic-scattering differential cross section becomes

$$
\begin{aligned}
\frac{d \sigma_{\text {elas }}}{d \Omega} & =\frac{1}{4} \lambda^{2}\left|1-\alpha e^{i \beta}\right|^{2}\left\{\sum_{l=0}^{l_{\max }}(2 l+1) P_{l}(\cos \theta)\right\}^{2} \\
& =\frac{1}{4} \lambda^{2}\left(1+\alpha^{2}-2 \alpha \cos \beta\right)\left\{\sum_{l=0}^{l_{\max }}(2 l+1) P_{l}(\cos \theta)\right\}^{2} .
\end{aligned}
$$

Unlike the case of the observables discussed earlier, the interpolation of this expression between "magic" values $k R=l_{\max }$ is not straightforward. This issue will be discussed in the following section. 
We may define a normalized angular distribution that describes only the angular dependence of the cross section by dividing by the angle-integrated elastic cross section. This yields

$$
\frac{1}{\sigma_{\text {elas }}} \frac{d \sigma_{\text {elas }}}{d \Omega}=\frac{1}{4 \pi} \frac{1}{(k R+1)^{2}}\left\{\sum_{l=0}^{l_{\max }}(2 l+1) P_{l}(\cos \theta)\right\}^{2} .
$$

As was the case for $\bar{\mu}$, this expression exhibits universality because the only dependence on nuclear parameters is in the radius $R$.

Finally, we note that the discussion in this section describes the Ramsauer approximation, but does not justify it. Discussions of the reasonableness of the approximations in the model and its range of applicability, as well as applications to neutron total cross sections and other observables, may be found in Refs. [2, 3, 4, 5, 6, 7, 8, 9]. 


\section{Interpolating the Ramsauer Scattering Am- plitude}

As previously noted, the Ramsauer approximation yields simple expressions for observables only when $k R$ is an integer equal to $l_{\max }$, the maximum value of angular momentum in the discrete sum resulting from applying a sharp-cutoff approximation to the scattering amplitude. The simple, intuitive interpolation procedure that is useful for angle-independent observables does not apply to the scattering amplitude and the angular distribution derived from it (except for the zero-degree quantities). In this section we devise a procedure for interpolating the scattering amplitude and study its applicability.

We define $l_{\max }$ as the nearest integer that is equal to or less than $k R$. We also define a deviation $\delta=k R-l_{\max }$, where $0 \leq \delta<1$.

To obtain an interpolable scattering amplitude, we choose to add an extra term to the Legendre expansion of the Ramsauer scattering amplitude of Eq. 23:

$$
f(\theta)=\frac{i}{2 k}\left(1-\alpha e^{i \beta}\right)\left\{\sum_{l=0}^{l_{\max }}(2 l+1) P_{l}(\cos \theta)+\xi e^{i \phi}\left(2 l_{\max }+3\right) P_{l_{\max }+1}(\cos \theta)\right\},
$$

where $\xi$ and $\phi$ are two real functions of the parameters in the problem. These functions provide sufficient flexibility to make some of the observables derived from the scattering amplitude coincide with those using the simple interpolation procedure of the last section (i.e. letting $k R$ be a continuous variable). It is not possible to find a pair of functions that yield agreement with the simple forms for all observables; this inconsistency is a limitation resulting from the sharp-cutoff approximation in the Ramsauer model, which is only slightly lifted by adding a single extra term to the scattering amplitude.

As an example, we will find forms for $\xi$ and $\phi$ that achieve consistency between the angular distribution and the simple interpolation procedure for two integral quantities, the angle-integrated elastic cross section and the average value of the cosine of the scattering angle. This example is particularly relevant to the use of Ramsauer-model angular distributions in transport calculations.

We first look for conditions on the functions $\xi$ and $\phi$ that provide consistency between the angle-integrated elastic scattering derived directly from Eq. 47 and the simply interpolated value

$$
\sigma_{\text {elas }}=\pi(R+\lambda)^{2}\left|1-\alpha e^{i \beta}\right|^{2}
$$

From the expression $\sigma_{\text {elas }}=\int d \Omega|f(\theta)|^{2}$ we find

$$
\begin{aligned}
\sigma_{\text {elas }} & =\pi \lambda^{2}\left|1-\alpha e^{i \beta}\right|^{2}\left\{\sum_{l=0}^{l_{\max }}(2 l+1)+\xi^{2}\left(2 l_{\text {max }}+3\right)\right\} \\
& =\pi \lambda^{2}\left|1-\alpha e^{i \beta}\right|^{2}\left\{\left(l_{\max }+1\right)^{2}+\xi^{2}\left(2 l_{\text {max }}+3\right)\right\} .
\end{aligned}
$$

We substitute $l_{\max }=k R-\delta$ in the last expression and rearrange it to get

$$
\sigma_{\text {elas }}=\pi \lambda^{2}\left|1-\alpha e^{i \beta}\right|^{2}\left\{(k R+1)^{2}-2 \delta(k R+1)+\delta^{2}+\xi^{2}(2 k R-2 \delta+3)\right\} .
$$


The desired result is achieved if the last three terms within the brackets cancel, which requires

$$
\xi^{2}=\frac{2 \delta(k R+1)-\delta^{2}}{2 k R-2 \delta+3}=\frac{2 \delta\left(l_{\max }+1+\delta\right)-\delta^{2}}{2 l_{\max }+3} .
$$

We thus find that the requirement that the scattering amplitude yield a smooth interpolation for the angle-integrated elastic cross section is satisfied by an appropriate choice for $\xi$. The other function, $\phi$, is not constrained by this requirement and is available for another purpose.

Next, we attempt to choose $\phi$ so that the average cosine of the scattering angle evaluated from Eq. 47 is given by $\bar{\mu}=1-(k R+1)^{-1}$, the result from the simple interpolation procedure. We follow the procedure in Sec. 2 beginning with Eq. 37, taking into account the extra term in the scattering amplitude. We get the correct expression by inserting the following values in Eq. 22 in Section 1:

$$
1-S_{l}= \begin{cases}1-\alpha e^{i \beta}, & 0 \leq l \leq l_{\max } \\ \xi e^{i \phi}\left(1-\alpha e^{i \beta}\right), & l=l_{\text {max }}+1 \\ 0, & l>l_{\max }+1\end{cases}
$$

Eq. 37 and the following development are replaced by

$$
\begin{aligned}
\sigma_{\text {elas }} \bar{\mu}= & \pi \lambda^{2}\left|1-\alpha e^{i \beta}\right|^{2}\left\{\left[\sum_{l=0}^{l_{\max }-1}(2 l+1)\right]+l_{\text {max }}+2 \xi \cos \phi\left(l_{\text {max }}+1\right)\right\} \\
= & \pi \lambda^{2}\left|1-\alpha e^{i \beta}\right|^{2}\left\{\left[\sum_{l=0}^{l_{\max }}(2 l+1)\right]-\left(l_{\text {max }}+1\right)+2 \xi \cos \phi\left(l_{\text {max }}+1\right)\right\} \quad(55) \\
= & \pi \lambda^{2}\left|1-\alpha e^{i \beta}\right|^{2}\left\{\left(l_{\max }+1\right)^{2}-\left(l_{\max }+1\right)+2 \xi \cos \phi\left(l_{\max }+1\right)\right\} \\
= & \pi \lambda^{2}\left|1-\alpha e^{i \beta}\right|^{2}\left\{(k R+1-\delta)^{2}-(k R+1-\delta)+2 \xi \cos \phi(k R+1-\delta)(57)\right. \\
= & \pi \lambda^{2}\left|1-\alpha e^{i \beta}\right|^{2}\left\{(k R+1)^{2}-(k R+1)\right. \\
& \left.-2 \delta(k R+1)+\delta^{2}+\delta+2 \xi \cos \phi(k R+1-\delta)\right\} \\
= & \pi(R+\lambda)^{2}\left|1-\alpha e^{i \beta}\right|^{2}\left\{1-\frac{1}{k R+1}\right. \\
& \left.+\frac{-2 \delta(k R+1)+\delta^{2}+\delta+2 \xi \cos \phi(k R+1-\delta)}{(k R+1)^{2}}\right\}
\end{aligned}
$$

The first two terms in the brackets of the last expression are the desired result, and to achieve it the third term must vanish. This condition allows us to determine $\cos \phi$ as

$$
\cos \phi=\frac{2 \delta(k R+1)-\delta^{2}-\delta}{2 \xi(k R+1-\delta)}=\frac{2 \delta l_{\max }+\delta^{2}+\delta}{2 \xi\left(l_{\max }+1\right)},
$$

where we use the previously found value of $\xi^{2}$ to determine $\xi$, taking the positive branch of the square root.

A very different result from the above example is obtained from the following form for the scattering amplitude, suggested by Dennis McNabb:

$$
f(\theta)=\frac{i}{2 k}\left(1-\alpha e^{i \beta}\right)\left\{\sum_{l=0}^{l_{\max }}(2 l+1) P_{l}(\cos \theta)+\left[(k R+1)^{2}-\left(l_{\max }+1\right)^{2}\right] P_{l_{\max }+1}(\cos \theta)\right\},
$$


which corresponds to the choices

$$
\xi=\left[(k R+1)^{2}-\left(l_{\max }+1\right)^{2}\right] /\left(2 l_{\max }+3\right)
$$

and $\phi=0$. This expression leads to a forward-scattering amplitude

$$
f(0)=\frac{i}{2 k}\left(1-\alpha e^{i \beta}\right)(k R+1)^{2},
$$

which is the same as the value obtained from simple interpolation of the results for integral $k R$. Therefore, this interpolation method for the scattering amplitude leads to smoothly-varying behavior for the forward scattering amplitude and quantities derived from it, which are the zero-degree differential cross section, the total cross section, and the Wick-limit approximation to the zero-degree cross section.

Figs. 1-4 show the effects of four different choices for $\xi$ and $\phi$ on angle-independent observables, and Figs. 5-8 show the corresponding angular distributions for several values of $k R$ between 4 and 4.75. The choices for $\xi$ and $\phi$ are:

- Method 1. No extra term added to the scattering amplitude; i.e. $\xi=0$ and $\phi=0$.

- Method 2. $\xi$ is chosen to yield a smoothly-varying angle-integrated cross section via Eq. 52, but the phase function $\phi$ is set to zero.

- Method 3. As in Method 2, $\xi$ is chosen to yield a smoothly-varying angleintegrated cross section via Eq. 52, and in addition the phase function $\phi$ is chosen to yield a smoothly-varying value for $\bar{\mu}$ according to Eq. 60 .

- Method 4. $\xi$ is chosen by Eq. 62 to yield smooth behavior for the zero-degree cross section; $\phi=0$.

The quantities shown in Figs. 1-4 are:

- Upper left: Elastic cross section from integration of the angular distribution divided by the simply-interpolated value Eq. 26.

- Upper right: The average value of the quantity $(1-\mu)$, where $\mu$ is the average cosine of the scattering angle.

- Center left: The zero-degree cross section divided by the simply-interpolated value of the elastic cross section Eq. 26.

- Center right: The fraction of the elastic scattering for angles greater than 90 degrees.

- Lower left: The value of $\xi$ (shown only when nonzero).

- Lower right: The value of $\phi$ (shown only when nonzero).

The figures show that Method 3 is the superior interpolation procedure for applications that require use of angular distributions. In addition to exactly reproducing the simple interpolation procedure for the elastic cross section and $1-\bar{\mu}$, this procedure yields a zero-degree differential cross section that is very close (but not identical) to the simply-interpolated value. The fraction of scattering beyond 90 degrees is not completely smooth, but yields better results than the other methods. 


\section{Acknowledgments}

This work was performed under the auspices of the U.S. Department of Energy by the University of California, Lawrence Livermore National Laboratory under contract No. W-7405-Eng-48. Looking into the effects of interpolating the Ramsauer-model angular distributions was stimulated by discussions with Dennis McNabb and John Anderson. 


\section{References}

[1] A. R. Edmonds, Angular Momentum in Quantum Mechanics (Princeton University Press, Princeton, 1960).

[2] J. M. Peterson, Phys. Rev. 125, 955 (1962).

[3] A. Bohr and B. Mottelson, Nuclear Structure, Vol. 1, p. 166 (Benjamin, New York, 1969).

[4] J. D. Anderson and S. M. Grimes, Phys. Rev. C 41, 2904 (1990).

[5] V. A. Madsen et al., Phys. Rev. C 56, 365 (1997).

[6] R. W. Bauer et al., Nucl. Sci. Eng. 130, 348 (1998).

[7] S. M. Grimes, J. D. Anderson, R. W. Bauer, and V. A. Madsen, Nucl. Sci. Eng. 130, 340 (1998).

[8] F. S. Dietrich, J. D. Anderson, R. W. Bauer, and S. M. Grimes, Phys. Rev. C 68, 064608 (2003).

[9] M. Azam and R. G. Gowda, Nucl. Sci. Eng. 144, 86 (2003). 

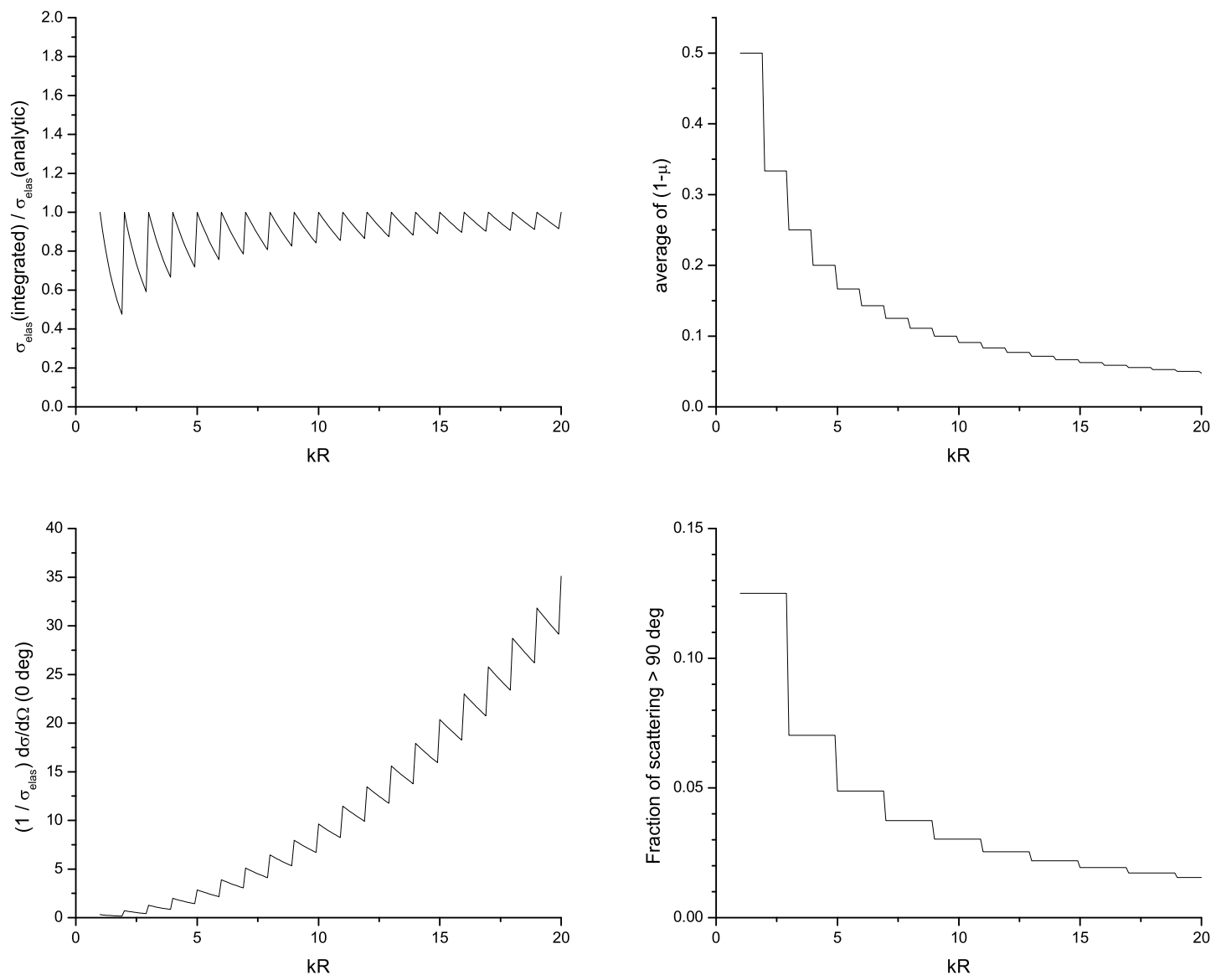

Figure 1: Method 1. Angular distribution calculated with no extra term; i.e. $\xi=0$ and $\phi=0$. 

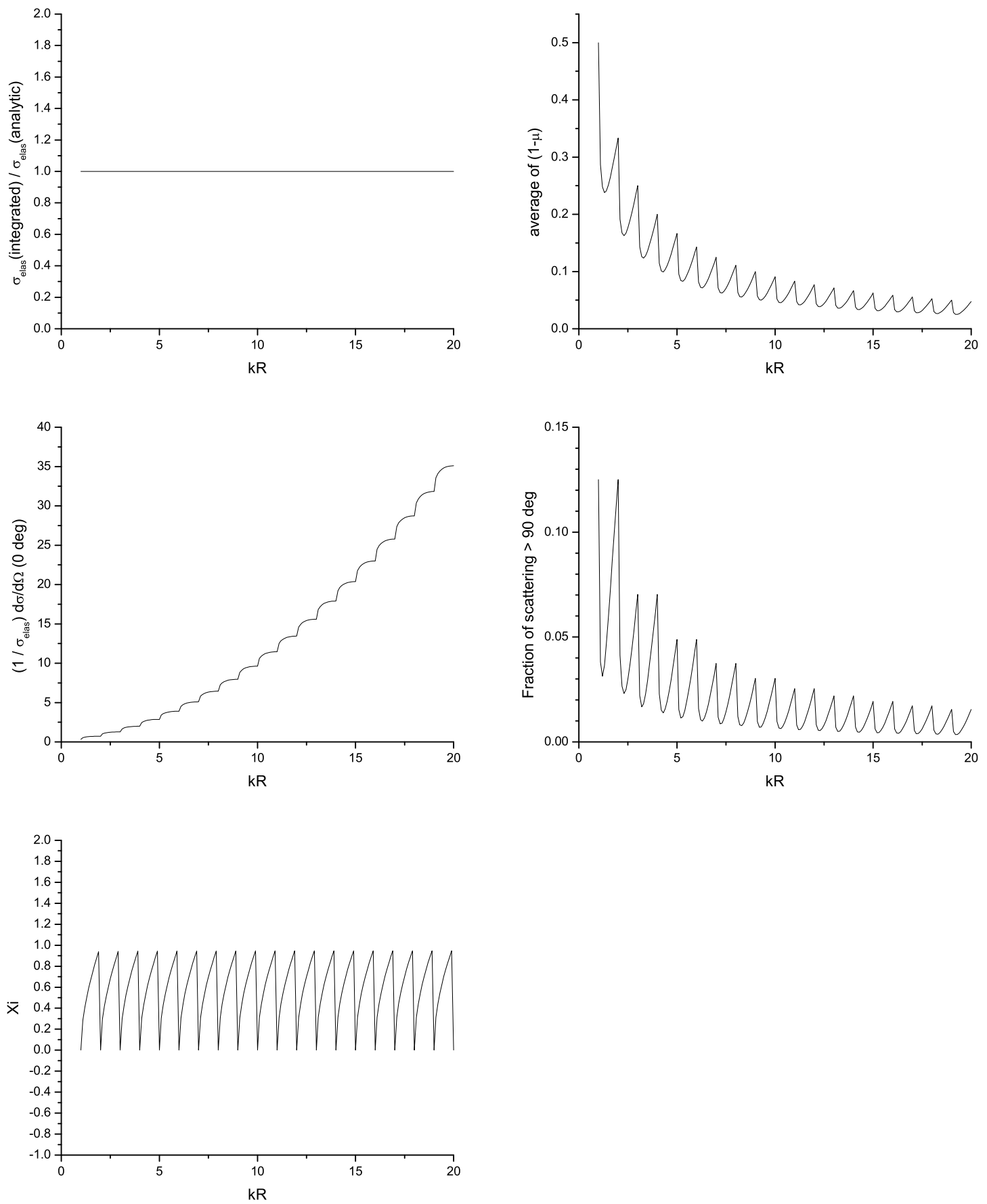

Figure 2: Method 2. Angular distribution calculated with $\xi$ chosen to yield smoothly-varying angle-integrated cross section and with $\phi=0$. 

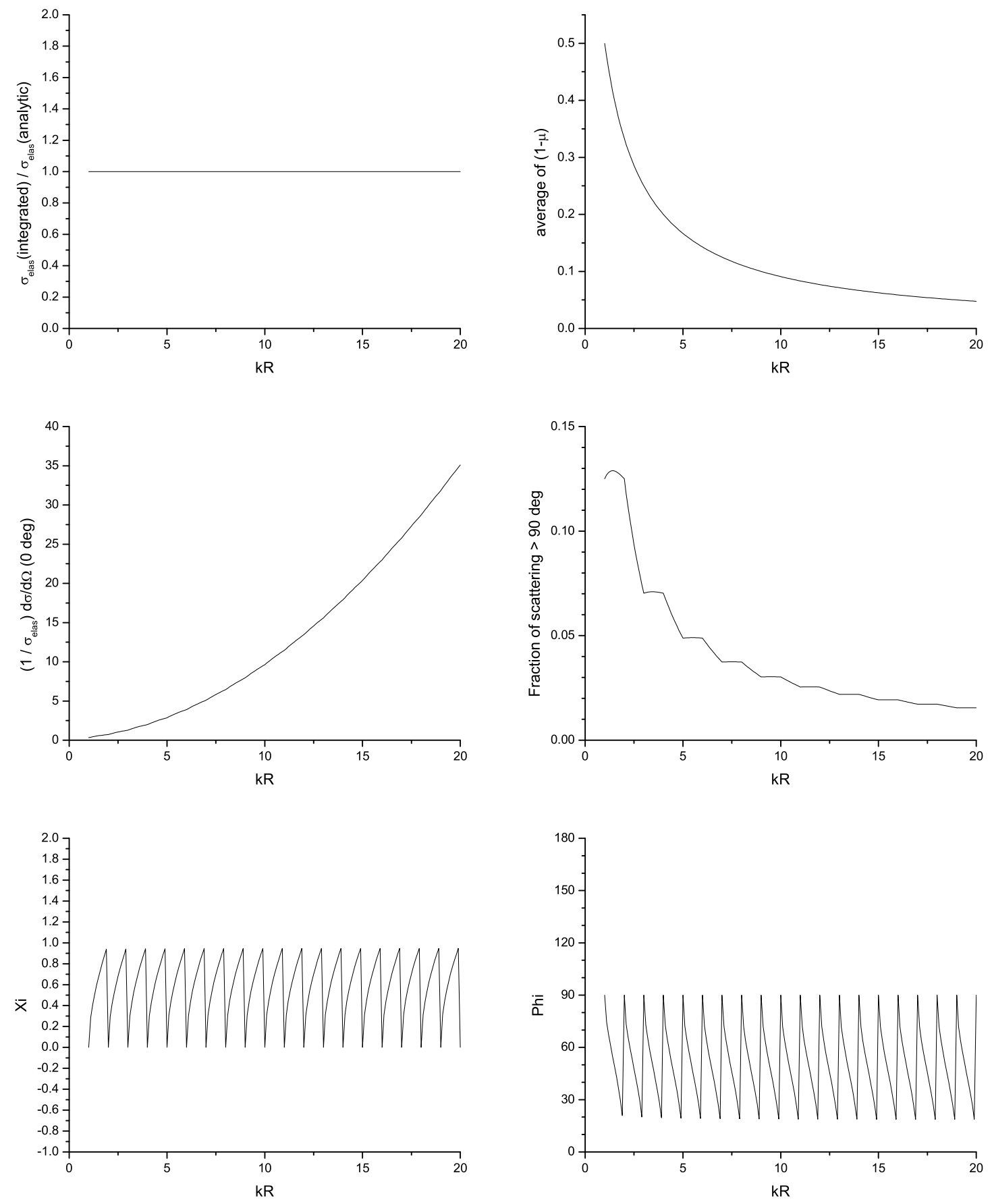

Figure 3: Method 3. Angular distribution calculated with $\xi$ chosen to yield smoothly-varying angle-integrated cross section and with $\phi$ chosen to yield smoothly-varying average cosine of scattering angle. 

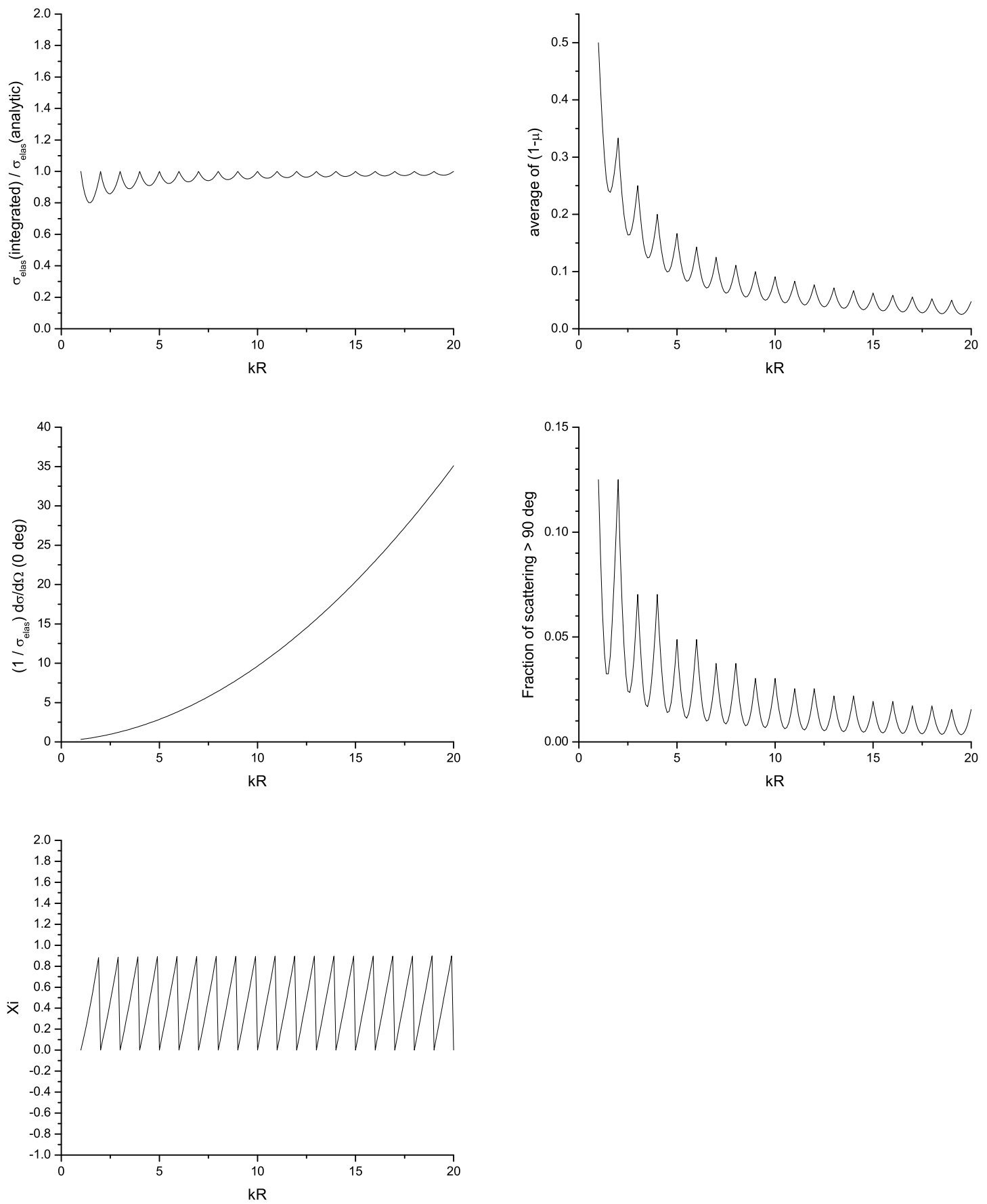

Figure 4: Method 4. Angular distribution calculated with $\xi$ chosen to yield smoothly-varying zero-degree differential cross section and with $\phi=0$. 


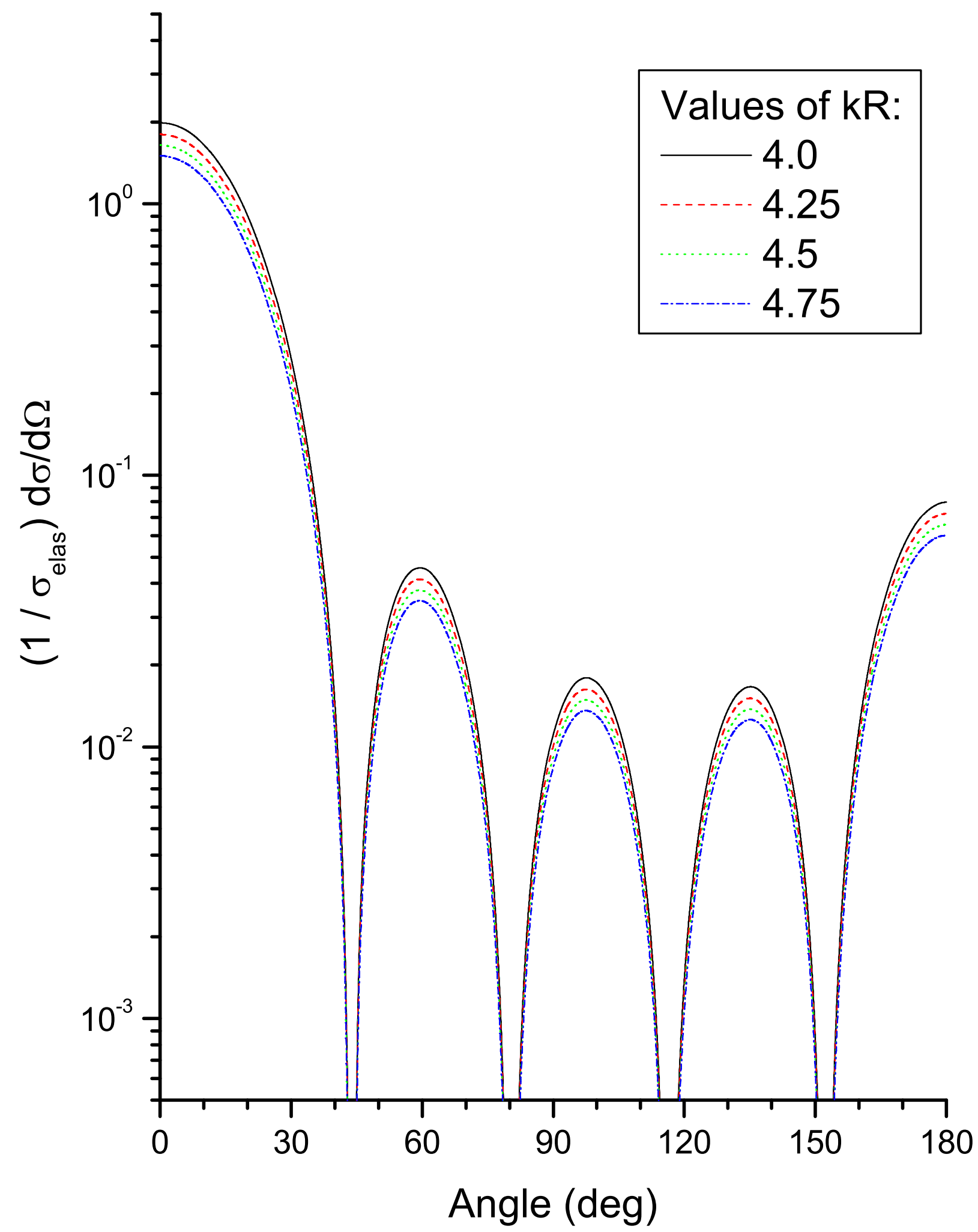

Figure 5: Method 1. Angular distribution calculated with no extra term; i.e. $\xi=0$ and $\phi=0$. 


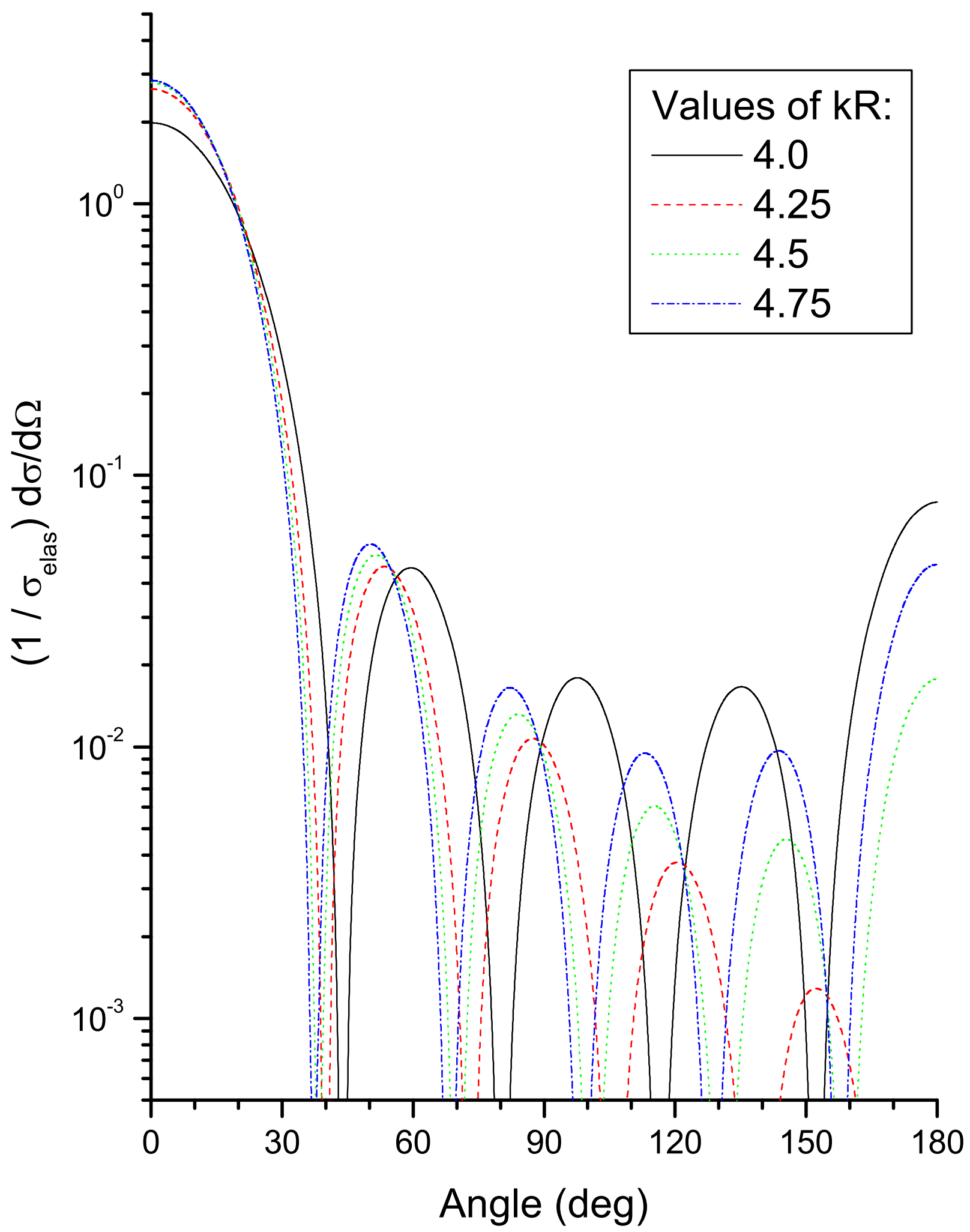

Figure 6: Method 2. Angular distribution calculated with $\xi$ chosen to yield smoothly-varying angle-integrated cross section and with $\phi=0$. 


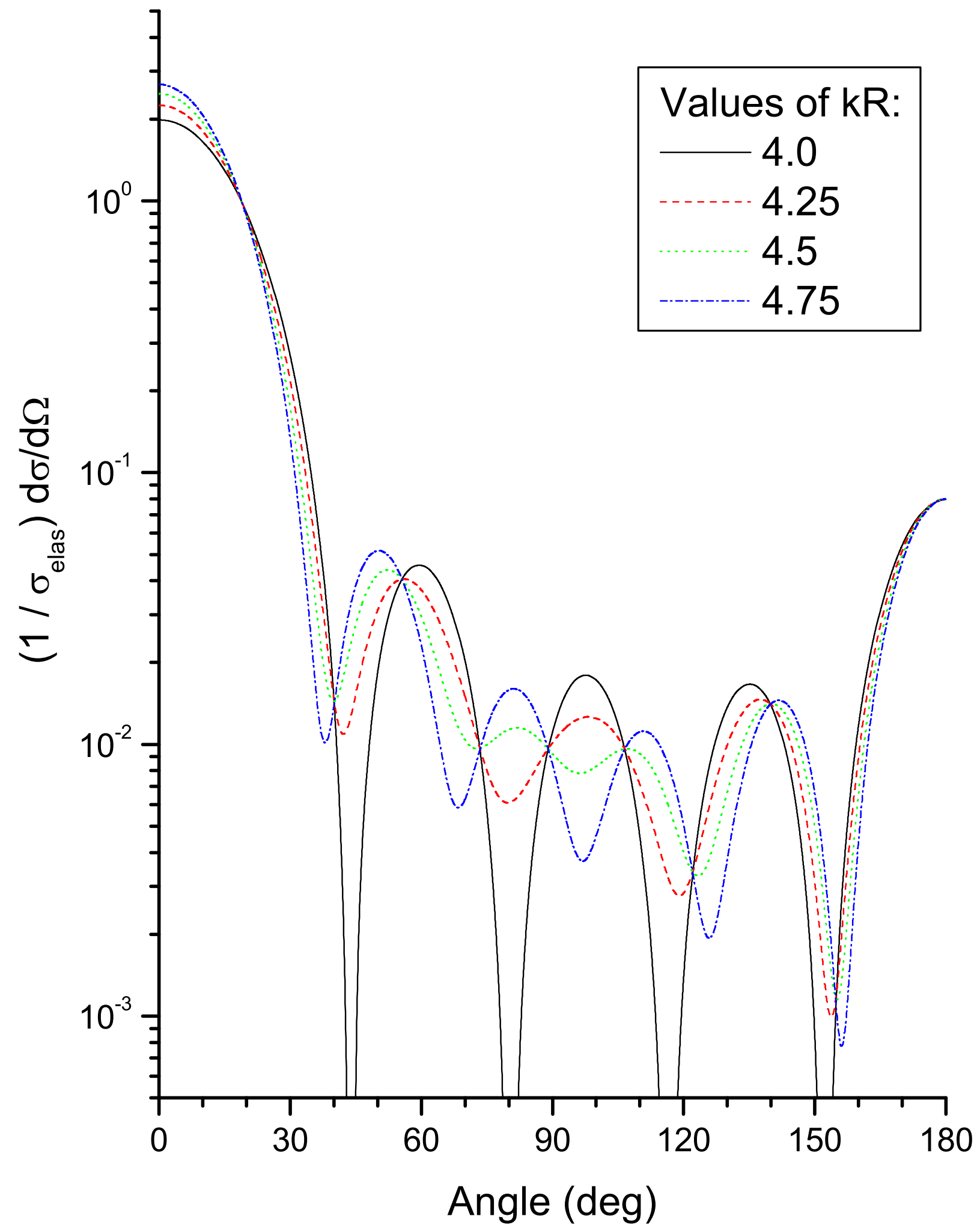

Figure 7: Method 3. Angular distribution calculated with $\xi$ chosen to yield smoothly-varying angle-integrated cross section and with $\phi$ chosen to yield smoothly-varying average cosine of scattering angle. 


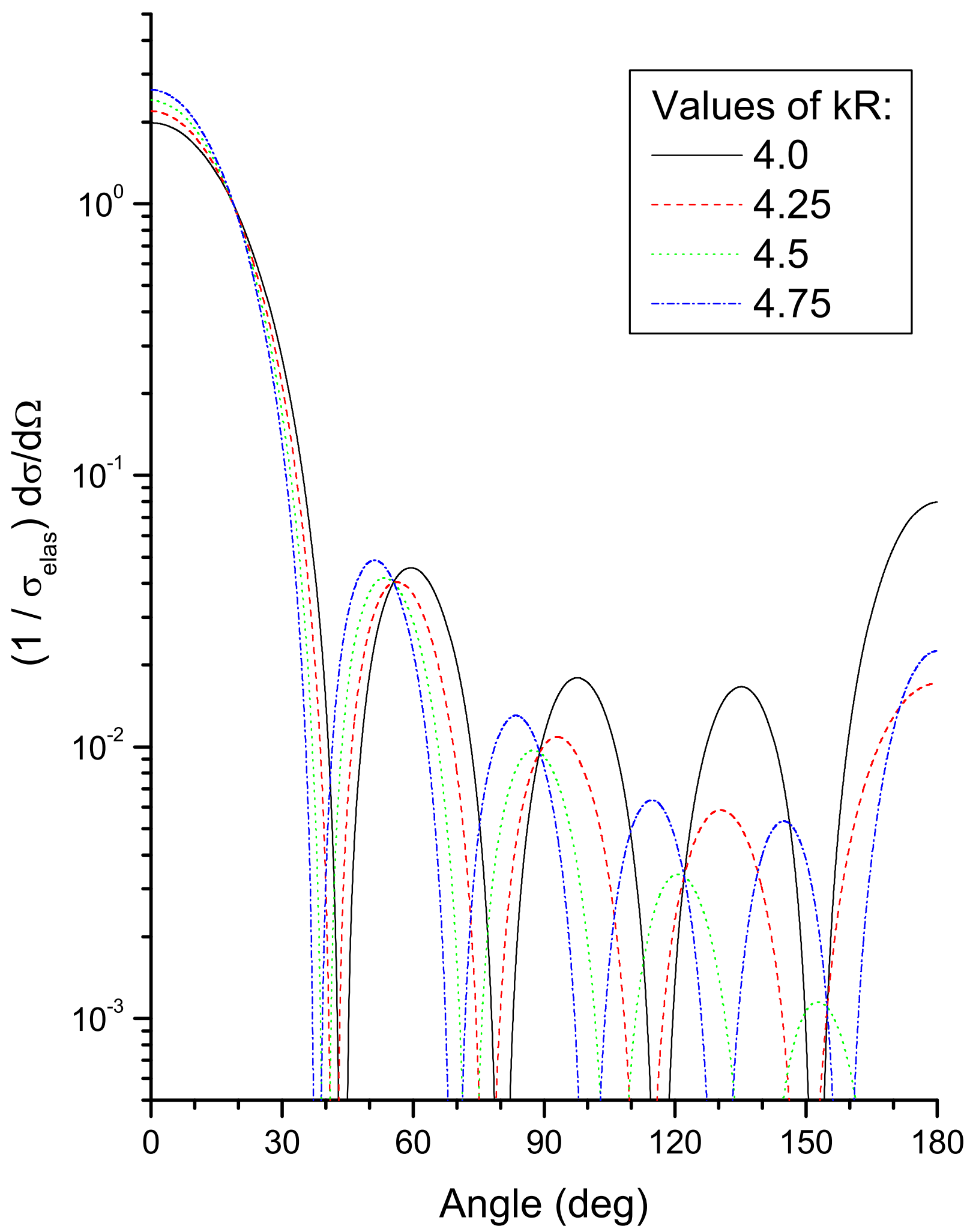

Figure 8: Method 4. Angular distribution calculated with $\xi$ chosen to yield smoothly-varying zero-degree differential cross section and with $\phi=0$. 